\title{
Zur Erschließung der Nutzung von Forschungsergebnissen durch Lehrpersonen. Forschungsrelevanz zwischen Theorie und Praxis
}

\author{
Jan-Hendrik Hinzke $\mathbb{D} \cdot$ Johanna Gesang $•$ Kris-Stephen Besa
}

Eingegangen: 14. November 2019 / Überarbeitet: 25. Juni 2020 / Angenommen: 14. Oktober 2020 /

Online publiziert: 5. November 2020

(C) Der/die Autor(en) 2020

Zusammenfassung Im deutschsprachigen Raum hat sich der Befund verfestigt, dass Lehrpersonen Forschungsergebnisse tendenziell als interessant einschätzen, diese aber kaum zur Änderung ihrer Unterrichtspraxis nutzen. Aus Sicht von Bildungspolitik, Bildungsadministration und Bildungsforschung, die einer Evidenzbasierung pädagogischer Praxis zuarbeiten, kann ein solcher Umgang mit Forschungsergebnissen nicht als gelungen bewertet werden. Der Beitrag präsentiert eine Möglichkeit, wie sich die Nutzung von Forschungsergebnissen durch Lehrpersonen vertiefend analysieren lässt. Dazu wurde in einer Interviewstudie die Differenz von normativem und theoretischem Anspruch einerseits und dessen praktischer Umsetzung andererseits untersucht. Aufgegriffen wurde dabei die Leitdifferenz der Praxeologischen Wissenssoziologie, diejenige zwischen propositionaler und performativer Logik. Empirisch zeigen sich diese Logiken als Theorien von Lehrpersonen über Forschungsergebnisse einerseits, als Habitus der Gestaltung unterrichtlicher Interaktion andererseits. Es wird exemplarisch dargelegt, wie bestimmte Relationen von Theorien und Habitus eine Relevanzsetzung von Forschungsergebnissen durch Lehrpersonen verhindern bzw. ermöglichen.

Dr. J.-H. Hinzke $(\bowtie)$

Wissenschaftliche Einrichtung Oberstufen-Kolleg, Fakultät für Erziehungswissenschaft, Universität Bielefeld, Universitätsstraße 23, 33615 Bielefeld, Deutschland

E-Mail: jan-hendrik.hinzke@uni-bielefeld.de

\section{Dr. J. Gesang}

Arbeitsgruppe Begabungsforschung/Individuelle Förderung, Institut für Erziehungswissenschaft, Westfälische Wilhelms-Universität Münster, Bispinghof 5/6, 48143 Münster, Deutschland

E-Mail: johanna.gesang@uni-muenster.de

Dr. K.-S. Besa

Arbeitsgruppe Allgemeine Didaktik und Unterrichtsforschung, Institut für Erziehungswissenschaft, Westfälische Wilhelms-Universität Münster, Bispinghof 5/6, 48143 Münster, Deutschland E-Mail: kbesa@uni-muenster.de 
Schlüsselwörter Nutzung von Forschungsergebnissen · Evidenzbasierung · Habitus · Lehrerforschung · Praxeologische Wissenssoziologie

\title{
Exploration of the use of research results by teachers. Relevance of research between theory and practice
}

\begin{abstract}
In German speaking countries, solid findings show that teachers tend to rate research results as interesting, but hardly use them to change their teaching practice. From the perspective of education policy, education administration and education research which support an evidence-based pedagogy, such a dealing with research results cannot be evaluated as successful. The contribution presents a way how to examine the use of research results by teachers by in-depth analysis. Therefore, an interview study was carried out considering the difference between normative and theoretical claim on the one hand and its practical realization on the other hand. The central difference of the praxeological sociology of knowledge-propositional vs. performative logic_was taken up. In the data, these logics show as theories of teachers about research results on the one hand, as habitus of designing interaction in teaching on the other hand. The results show, how certain relations of theories and habitus prevent or allow that research results become relevant for teachers.
\end{abstract}

Keywords Evidence-based approach $\cdot$ Habitus · Praxeological sociology of knowledge $\cdot$ Teacher research $\cdot$ Use of research

Die Anforderung, sich mit Forschungsergebnissen bzw. Evidenzen auseinanderzusetzen, wird von der deutschen Bildungspolitik seit der Jahrtausendwende verstärkt an den LehrerInnenberuf herangetragen (vgl. Hartmann et al. 2016, S. $180 \mathrm{f}$.). Damit folgt die Bildungspolitik dem Steuerungsmodell der Evidenzbasierung (vgl. Zlatkin-Troitschanskaia 2016, S. 6), das seinen Durchbruch mit dem Aufkommen großer internationaler Schulleistungsstudien (v. a. TIMSS, PISA, IGLU) erhielt. Auf deren Ergebnisse wurde mit der Umsetzung einer neuen Steuerungspolitik reagiert, die auf Generierung von Forschungsdaten und deren Rückmeldung an verschiedene Bereiche im Bildungssystem setzt. Bildungsstandards, Lernstandserhebungen und Vergleichsarbeiten, zentrale Abschlussarbeiten und Schulinspektionen sind Kennzeichen einer evidenzbasierten Steuerungspolitik im Schulsystem, die in Deutschland auf Ebene der Kultusministerkonferenz (KMK) betrieben und von den Bundesländern - auf unterschiedliche Weise - umgesetzt wird (vgl. Thiel et al. 2019).

Es deutet sich hier bereits an, dass die Begriffe ,Forschungsergebnisse“ und ,Evidenz‘ im Diskurs um Evidenzbasierung im Bildungssystem insgesamt betrachtet nicht systematisch voneinander getrennt werden (s. Abschn. 1). Vor diesem Hintergrund werden die beiden Begriffe in den ersten drei Abschnitten dieses Beitrags immer wieder bewusst zusammen genannt.

Mittlerweile liegen mehrere Studien dazu vor, wie die Anforderung, Forschungsergebnisse bzw. Evidenzen für die eigene Praxis zu nutzen, von Lehrpersonen aufgegriffen wird (vgl. etwa Demski 2017; Zlatkin-Troitschanskaia et al. 2016). Dabei 
zeigt sich, dass Lehrpersonen Forschungsergebnissen bzw. Evidenzen zwar oftmals offen gegenüberstehen, aber nur in Ausnahmefällen zur Richtschnur ihres Handelns machen - ein Umstand, der als partielles Scheitern evidenzbasierter Bildungspolitik gedeutet werden kann.

Der Beitrag stellt eine Studie vor, die diese Befundlage zum Ausgangspunkt genommen und sich zum Ziel gesetzt hat, das Phänomen der Nutzung von Forschungsergebnissen durch Lehrpersonen näher zu verstehen. Dabei wurde - anders als im Mainstream der Studien zum Thema - rekonstruktiv vorgegangen, was ermöglichte, nicht nur kommunikatives, sondern auch implizites, handlungsleitendes Wissen von Lehrpersonen zu erfassen. Entsprechend wurde die Forschungsfrage verfolgt, welche Bedeutung kommunikativen und handlungsleitenden Wissensbeständen von Lehrpersonen bei der Nutzung von Forschungsergebnissen zukommt.

Auf eine Charakterisierung der Forschungs- und Evidenzbasierung im Bildungssystem (Abschn. 1) und einen Verweis auf Studien zur Nutzung von Forschungsergebnissen und Evidenzen durch Lehrpersonen (Abschn. 2) folgt eine praxeologischwissenssoziologische Perspektivierung (Abschn. 3). Dieser liegt eine Interviewstudie mit Lehrpersonen zugrunde, deren Durchführung (Abschn. 4) und Ergebnisse (Abschn. 5) im Anschluss vorgestellt werden. Abschließend werden die Ergebnisse ebenso diskutiert wie das Potenzial und die Grenzen der Studie (Abschn. 6).

\section{Forschungs- und Evidenzbasierung im Bildungssystem: Forcierung durch Bildungspolitik, Bildungsadministration und (Teile der) Bildungsforschung}

Die von der KMK forcierte Forschungs- und Evidenzbasierung im Bildungssystem kommt prominent in der aktualisierten Gesamtstrategie der Kultusministerkonferenz zum Bildungsmonitoring zum Ausdruck. Forschungsprojekte sollen darüber Auskunft geben, ,inwieweit die Implementation der Bildungsstandards und der Maßnahmen der Qualitätsentwicklung und Qualitätssicherung in die schulische Praxis gelungen ist, welche Gelingensbedingungen dafür förderlich sind und welcher Handlungsbedarf besteht“ (KMK 2016, S. 12). Ziel ist es, „mehr anwendungsbezogenes Wissen für Bildungspolitik und pädagogische Praxis“ (KMK 2016, S. 15) zu generieren, das über Transfer- und Implementationsstrategien verbreitet und u. a. ,für die Weiterentwicklung der Unterrichtspraxis“ (KMK 2016, S. 15) genutzt werden soll. Schulen werden hier als Akteure adressiert, die datenbasierte Qualitätssicherung und -entwicklung betreiben sollen. Dieses Vorgehen wird explizit als „Grundlage für eine evidenzbasierte Bildungspolitik in allen Ländern“ (KMK 2016, S. 3) bezeichnet.

Dass die Länder diese Bildungspolitik umsetzen, wird an dem Positionspapier der Landesinstitute und Qualitätseinrichtungen der Länder zum Transfer von Forschungswissen deutlich. Dabei positionieren sich die Landesinstitute und Qualitätseinrichtungen (2018) als Mittler zwischen Wissenschaft und bildungspolitischen, bildungsadministrativen und schulischen Perspektiven, indem sie zwei „Richtungen“ von „Transferbemühungen“ unterscheiden: Im Sinne einer „Angebotsorientierung“ werden „wissenschaftliche Erkenntnisse“ bzw. „relevantes Forschungswissen“ recherchiert, geprüft und ggf. in verschiedene Handlungsbereiche des Schulsystems 
„eingespeist“ (Landesinstitute und Qualitätseinrichtungen 2018, S. 9). Im Sinne einer „Bedarfsorientierung“ werden „Forschungs- und Unterstützungsbedarfe“ aus diesen Handlungsbereichen an die „Wissenschaft“ (Landesinstitute und Qualitätseinrichtungen 2018, S. 9) rückgekoppelt.

Auffällig ist bei beiden Veröffentlichungen, dass sie keine klaren Definitionen von Forschung bzw. Forschungsergebnissen und Evidenz bieten. In ihrer Gesamtstrategie scheint die KMK diese Begriffe nahezu synonym zu verwenden, im Positionspapier werden wissenschaftliche Erkenntnisse und Forschungswissen in einer Linie genannt. Hierin drückt sich eine Diffusität der Begriffe aus, die im Fall von Evidenz z.T. aus der Übersetzung aus der englischsprachigen Debatte hervorgeht (vgl. etwa Demski 2017, S. 24). Ein breites Evidenz-Verständnis reicht von „wissenschaftlich-empirischen Studien bis hin zu kollegialen Hospitationen und Schülerfeedbacks" (van Ackeren et al. 2013, S. 54; vgl. Demski 2017). Etwas enger ist ein Verständnis, nach dem sich die beiden Evidenzquellen ,wissenschaftliches Wissen “ und Wissen aus , evidenzbasierten Steuerungsinstrumenten " wie Lernstandserhebungen und Schulinspektionen unterscheiden lassen (vgl. Altrichter 2019, S. 27). Wenn Altrichter und Maag Merki (2016, S. 21) zugleich darlegen, dass Steuerungswissen „durch wissenschaftlichen Sachverstand unterstützt“" generiert wird, erscheint es allerdings weiterhin schwierig, wissenschaftliches Wissen bzw. Forschungsergebnisse von einem Wissen, das durch Steuerungsinstrumente generiert wurde, eindeutig abzugrenzen. Dies zeigt sich beispielsweise auch mit Blick auf das vom Bundesministerium für Bildung und Forschung (BMBF) geförderte Verbundprojekt EviS, in dem evidenzbasiertes Handeln einerseits beforscht wird und die ForscherInnen andererseits angeben, durch die Befunde ,ein wissenschaftlich fundiertes evidenzbasiertes Steuerungswissen bereit[zu]stellen“, das „,zur Förderung einer evidenzbasierten administrativen und pädagogischen Schulpraxis beiträgt" (Zlatkin-Troitschanskaia et al. 2016, S. 10).

Dass Forschungs- und Evidenzbasierung sowohl von der Bildungspolitik und den Landesinstituten als auch von Teilen der Bildungsforschung gefördert wird, lässt sich als Faktor deuten, der die Anforderung an Schulen und Lehrpersonen, sich mit Forschungsergebnissen bzw. Evidenzen auseinanderzusetzen, verstärkt. So führt die Überprüfung der Erreichung vorgegebener Ziele (z. B. in Form von Bildungsstandards und Qualitätsrahmen) sowie die Rückmeldung der Ergebnisse zu der Erwartung, dass die schulischen AkteurInnen diese aufnehmen, umsetzen und dadurch zielförderliche Entwicklungen an der Schule einsetzen (vgl. Altrichter und Maag Merki 2016, S. 21). Dem Programm der Evidenzbasierung kommen nicht nur Funktionen des Bildungsmonitorings und der Unterstützung politischer Entscheidungsfindung zu, sondern explizit wird auch das Ziel verfolgt, ,das Handeln der schulischen Akteure durch extern bereitgestellte Daten [...] effektiver und effizienter zu gestalten und so zur Entwicklung von Schule und Unterricht beizutragen“ (Demski et al. 2016, S. 39). Damit sind nicht nur Schulleitungen adressiert. Evidenz soll auch Lehrpersonen als „Problembewusstsein, als Leitfaden und Hilfestellung, als Ergänzung und Korrektiv“ (Bauer et al. 2015, S. 190) dienen - als ein Korrektiv, ,das einen Qualitätsgewinn im professionellen Handeln verspricht und dieses rational begründen kann“ (Bauer et al. 2015, S. 190). 
Zwar gilt das deutsche Schulsystem als low stakes-System (vgl. van Ackeren et al. 2017, S. 244), doch lässt sich zusammenfassend auch für den deutschsprachigen Kontext konstatieren, dass der Erwartungsdruck an Schulleitungen und Lehrpersonen bezüglich einer evidenzbasierten Weiterentwicklung und Rechtfertigung ihrer Praxis gestiegen ist (vgl. Bonsen und Berkemeyer 2014, S. 920; Thiel et al. 2019, S. 316f.). Im Rahmen erweiterter Schulautonomie können diese AkteurInnen eigenständig Entscheidungen treffen, doch werden sie zugleich im Sinne einer accountabilityPolitik als verantwortlich für ihren Erfolg und Misserfolg angesehen (vgl. Altrichter et al. 2016, S. 114f.).

\section{Forschung zur Nutzung von Forschungsergebnissen und Evidenzen durch Lehrpersonen im deutschsprachigen Raum}

Bereits 2011 resümierte Terhart bei einer Sichtung der Forschung zum Umgang von Lehrpersonen mit neueren Steuerungsinstrumenten, dass ,keineswegs von einer reibungslosen Einführung und Umsetzung, sondern allenfalls von einer gewissen Öffnung [...] auf einer allgemeinen, eher deklamatorischen Ebene die Rede sein kann“. Und weiter: „Als Auslöser für konkrete Prozesse der Unterrichtsentwicklung wirken sie insgesamt gesehen jedoch noch nicht" (Terhart 2011, S. 214f.). Dieses Resümee weist in eine ähnliche Richtung wie die Befunde von Bach et al. (2014, S. 64), die konstatieren, dass vorliegende Studien ,,von einer grundsätzlich hohen Bereitschaft der Lehrkräfte, sich mit den Ergebnissen von Vergleichsarbeiten und standardbasierten Tests auseinanderzusetzen" berichten, jedoch auch deutlich wird, dass aus den „,Tests abgeleitete Maßnahmen [...] mehrheitlich nicht über einzelne Klassen“ (Bach et al. 2014, S. 64) hinausgehen.

Aktuellere Veröffentlichungen kommen zu einem ähnlichen Fazit. So berichten etwa Altrichter et al. (2016, S. 250), dass ,ein relevanter Teil der Lehrpersonen [...] Bereitschaft, sich mit den Ergebnissen von Lernstandserhebungen auseinanderzusetzen und Konsequenzen abzuleiten“, zeigt. Allerdings hätten die „meisten Studien enttäuschend wenig Nutzung von Datenrückmeldung für Unterrichtsentwicklung erhoben“ (Altrichter et al. 2016, S. 250f.). In der Studie von Wurster et al. (2017) gibt die Mehrheit der Lehrpersonen an, ,, auf Basis von Evaluationsergebnissen Unterrichtsentwicklung zu betreiben", doch variieren die Angaben unter den Befragten erheblich und es wird erkennbar, dass es sich eher um ,einzelne Maßnahmen“ denn um eine „mehrere Aktivitäten umfassende Nutzung“ (Wurster et al. 2017, S. 645) handelt.

Im BMBF-geförderten Forschungsschwerpunkt Steuerung im Bildungssystem (SteBiS) wurde u.a. die Nutzung von Test- und Prüfungsergebnissen für evidenzbasiertes Handeln untersucht. Es zeigt sich, dass die befragten Lehrpersonen eine Orientierung an externen Evidenzen an ihren Schulen (etwa Schulinspektion) wesentlich geringer ausgeprägt sehen als die Orientierung an schulinternen Evidenzen (etwa Schülerfeedbacks) (vgl. Zlatkin-Troitschanskaia et al. 2016, S. 19, 23f.). Hinsichtlich der Nutzung von Vergleichsarbeiten zur datenbasierten Schulentwicklung wird zudem in einer qualitativen Interviewstudie deutlich, dass das Nutzungsniveau eher gering und eine systematische schulinterne Kommunikation selten ist 
(vgl. Kuper et al. 2016, S. 41, 51). Dies weist in eine ähnliche Richtung wie die Fragebogenstudie von Demski (2017), in der auf Individualebene drei Cluster identifiziert wurden, die sich hinsichtlich des Ausmaßes der Nutzung verschiedener Evidenzquellen unterscheiden. Dem Cluster mit einer überdurchschnittlichen Nutzung werden dabei mit Abstand am wenigsten Lehrpersonen zugerechnet (vgl. Demski 2017, S. 231 ff.). Aus einer aktuellen Fragebogenstudie von Rochnia und Trempler (2019) geht zudem hervor, dass Lehrpersonen bildungswissenschaftliche Forschungsresultate nachrangig gegenüber anderen externen Wissensquellen rezipieren.

In ihrer Interviewstudie zieht Demski (2017) das Fazit, dass die Lehrpersonen „vergleichsweise wenige und kaum konkrete Beispiele der Nutzung von evidenzbasierten Wissensbeständen“ (Demski 2017, S. 304) anführen - ein Befund, der auch aus der Interviewstudie von Hetmanek et al. (2015) hervorgeht. Die Lehrpersonen nannten zwar bildungswissenschaftliche Forschungsresultate ,,vergleichsweise häufig als Ressource“ (Hetmanek et al. 2015, S. 204) für ihre tägliche Arbeit, wandten diese allerdings „kaum im Sinne einer evidenzbasierten Praxis“ (Hetmanek et al. 2015, S. 193) an.

Zusammenfassend zeigt sich ein relativ einheitliches Bild: Es wird eine Nutzung von Forschungsergebnissen und Evidenzen konstatiert, die - gemessen an den Ansprüchen der Forschungs- und Evidenzbasierung (s. Abschn. 1) - als ernüchternd $\mathrm{zu}$ bezeichnen ist. Einerseits scheint bei einem Teil von Lehrpersonen Interesse und Bereitschaft vorhanden zu sein, sich mit Forschungsergebnissen und Evidenzen auseinanderzusetzen. Andererseits wird erkennbar, dass Lehrpersonen Forschungsergebnisse und Evidenzen selektiv wahrnehmen und verhältnismäßig selten zur Veränderung der eigenen (Unterrichts-)Praxis nutzen. Wenn es zur Unterrichtsentwicklung kommt, dann werden eher kleinere Anpassungen als grundlegende Veränderungen vorgenommen.

In forschungsmethodischer Hinsicht fällt auf, dass beinahe durchgehend Selbstauskünfte eingeholt wurden. Dabei wird von manchen der angeführten AutorInnen kritisch angemerkt, dass die Nutzung von Forschungsergebnissen und Evidenzen ein sensibles Thema darstellt, wodurch sich Fragen nach der sozialen Erwünschtheit von Antworten stellen (vgl. Altrichter et al. 2016, S. 249; Demski 2017, S. 208; Zlatkin-Troitschanskaia et al. 2016, S. 30). In Folge dessen könnten die dargelegten Ergebnisse tendenziell sogar zu positiv ausfallen.

\section{Praxeologisch-wissenssoziologische Perspektivierung}

In dieser Studie soll mittels einer praxeologisch-wissenssoziologischen Perspektivierung eine Möglichkeit vorgestellt werden, über den in Selbstauskünften transportierten theoretischen Gehalt von Aussagen hinausgehend Aspekte der beruflichen Praxis von Lehrpersonen näher zu analysieren.

Die Praxeologische Wissenssoziologie wurde von Bohnsack auf Basis der Interpretation verschiedener Datensorten (insb. Gruppendiskussionen, Interviews, Videos und Fotos) entwickelt. Grundlegend ist die Annahme, dass im Handeln sozialer AkteurInnen zwei Logiken wirksam werden. Die propositionale Logik bezeichnet die 
„Logik der Theoriekonstruktion“ (Bohnsack 2017, S. 17). Sie dokumentiert sich im Datenmaterial als Orientierungsschemata, d.h. als Ausdruck ,kommunikativen “ Wissens (vgl. Bohnsack 2017, S. 103). In der in diesem Beitrag dargelegten Studie haben sich hinsichtlich der propositionalen Logik zum einen Common SenseTheorien von Lehrpersonen als relevant erwiesen, die mit Bohnsack (2017, S. 326) als „Theorien“ bestimmt werden, ,welche die Erforschten [...] über die eigene und auch die fremde Handlungspraxis entwerfen". Zum anderen wurden von Lehrpersonen wahrgenommene Normen rekonstruiert, die mit Erwartungen verbunden sind, die sich auf das Handeln in einem sozialen Gefüge beziehen (vgl. Bohnsack 2017, S. 103f.). Der propositionalen Logik konstitutionslogisch voraus geht die performative Logik. Sie dokumentiert sich als Orientierungsrahmen, d.h. als Ausdruck ,konjunktiven“ Wissens, welches die Form von Habitus als Struktur der Praxis annehmen kann (vgl. Bohnsack 2017, S. 54). In diesem Beitrag wird ein HabitusBegriff für die Interpretation genutzt, der sich ,von den starken gesellschaftstheoretischen Vor-Annahmen der Bourdieu'schen Analyse“ (Bohnsack 2017, S. 26) löst und stattdessen einen rekonstruktiven Zugang zu handlungsleitenden Wissensbeständen von Lehrpersonen ermöglicht. Habitus wird dabei primär als „strukturierende Struktur“ (Bohnsack 2017, S. 299), als „Modus Operandi“ (Bohnsack 2017, S. 51) in den Blick genommen und dabei - mit Bohnsack (2017, S. 179) - weitgehend synonym mit Orientierungsrahmen genutzt. Der Fokus liegt auf berufsbezogenen, kollektiven Habitus, in denen sich von mehreren Lehrpersonen geteiltes ,konjunktives', d. h. implizites und handlungsleitendes Wissen ausdrückt (vgl. Bohnsack 2017, S. $102 \mathrm{ff}$.).

Propositionale und performative Logik sind beide Bestandteil von Alltagserfahrungen, befinden sich aber in einer ,,notorischen Diskrepanz“ (Bohnsack 2017, S. 54) zueinander, da sie unterschiedliche Zugänge zur Welt eröffnen. Einem theoretisierenden Zugang (propositionale Logik) steht ein praktischer Zugang (performative Logik) gegenüber. Für die Beforschung der Nutzung von Forschungsergebnissen und Evidenzen folgt daraus, dass es lohnenswert sein könnte, sowohl berufsbezogene Habitus von Lehrpersonen als Strukturen der Praxis als auch deren Relationen zu den von Lehrpersonen wahrgenommenen Common Sense-Theorien und Normen zu rekonstruieren.

\section{Forschungsfrage und Forschungsdesign}

Das von einem Forschungsfonds an der Universität Bielefeld geförderte Projekt „Nutzung von Bildungsforschung durch Lehrpersonen“ (NuBiL) (2018-2019) verfolgte die Frage, wie Lehrpersonen Ergebnisse der Bildungsforschung in ihrem Berufsalltag verwenden. Dieser Frage sollte im Rahmen sowohl einer Fragebogen- als auch einer Interview-Teilstudie nachgegangen werden (vgl. für erste Ergebnisse Otto et al. 2019). Nach Sichtung des Diskurses um Forschungs- und Evidenzbasierung (s. Abschn. 1) wurde die Fragestellung für die Interview-Teilstudie insofern adaptiert, als nun nicht die Nutzung von Ergebnissen der Bildungsforschung, sondern von Forschungsergebnissen allgemein im Mittelpunkt stand. Dabei wurde grundsätzlich Bromme et al. (2014, S. 5) gefolgt, nach denen Forschung Daten hervorbringt, die 
erst „durch eine entsprechende Interpretation [...] zu ,Evidenz ‘ für Einschätzungen oder Entscheidungen“" werden. Forschung wurde somit als eine mögliche Grundlage für evidenzbasiertes Handeln betrachtet. Auf eine enggeführte Definition von Forschung bzw. Forschungsergebnissen wurde indes verzichtet.

Die Studienlage (s. Abschn. 2) lässt erkennen, dass die Nutzung von Forschungsergebnissen bislang vor allem standardisiert oder inhaltsanalytisch über Selbstauskünfte erforscht wurde. Während dadurch kommunikatives Wissen von Lehrpersonen erfasst wurde, blieb deren implizites, handlungsleitendes Wissen (s. Abschn. 3) unberücksichtigt. Um das Phänomen der Nutzung von Forschungsergebnissen näher zu analysieren, befasst sich die hier vorgestellte Interview-Teilstudie mit folgender Forschungsfrage: Welche Bedeutung kommen kommunikativen und handlungsleitenden Wissensbeständen von Lehrpersonen bei der Nutzung von Forschungsergebnissen zu?

Dieser Frage wurde nachgegangen, indem an fünf Schulen - zwei Grundschulen, drei Gymnasien - zweier deutscher Bundesländer in einem Querschnittsdesign 19 episodische Interviews mit Lehrpersonen geführt wurden. Episodische Interviews bieten das Potenzial, sowohl semantisches Wissen in Form von Argumentationen als auch narrativ-episodisches Wissen in Form von Erzählungen selbsterlebter Ereignisse hervorzubringen (vgl. Flick 2017). Dadurch erschien diese Interviewform geeignet, Daten zu generieren, in denen sich kommunikative wie auch handlungsleitende Wissensbestände von Lehrpersonen zeigen. Ein Interviewleitfaden, der bei den Erhebungen flexibel gehandhabt und um immanente Nachfragen ergänzt wurde, sollte dazu dienen, die Vergleichbarkeit der Interviews zu erhöhen (vgl. Nohl 2017, S. 17f.). Das Sampling sollte ermöglichen, über Kontrastierungen hinsichtlich Schulformen im allgemeinbildenden Sektor (Primar- vs. Sekundarstufe I und II) und Bundesländer (Flächenland vs. Stadtstaat) ein mögliches Spektrum an Ergebnissen zu erhalten. Die Auswahl der pro Schule interviewten drei bzw. vier Lehrpersonen erfolgte über die per E-Mail angeschriebenen Schulleitungen. Mögliche Selektionseffekte sind hier wahrscheinlich, sei es über eine Positivauswahl durch die Schulleitungen, sei es über die Motivation der Lehrpersonen.

Den Lehrpersonen wurde angekündigt, dass der Umgang mit beruflichen Alltagssituationen im Zentrum des Interesses steht. Angesichts der im Forschungsstand aufscheinenden mäßigen Relevanz von Forschungsergebnissen für die Gestaltung der beruflichen Praxis durch Lehrpersonen (s. Abschn. 2) wurde der thematische Fokus von den InterviewerInnen anfangs bewusst nicht auf Forschung gelegt. Stattdessen wurden die Befragten eingangs gebeten, darzulegen, was sie einer außenstehenden Person über ihre Arbeit an der Schule erzählen würden. Diese Frage sollte als relativ offener Impuls Erzählungen und Beschreibungen über die eigene Berufspraxis evozieren. Daraufhin wurden die Lehrpersonen gebeten, Unterrichtssituationen der zurückliegenden zwei Wochen zu schildern, die ihnen in Erinnerung geblieben sind. Das Gesprächsthema wurde durch diese Frage insofern enger gefasst, als nun auf den Teilbereich Unterricht und das unterrichtliche Handeln der Lehrpersonen fokussiert wurde. Gleichsam war auch diese Frage relativ offen formuliert, sie sollte insbesondere narrativ-episodisches Wissen zum Vorschein bringen. Erst in einem hinteren Leitfadenteil wurden die Lehrpersonen gebeten zu erzählen, wann und wie sie erstmalig mit Forschung in Kontakt gekommen sind, wie sich der Kontakt im 
beruflichen Alltagshandeln entwickelt hat und welche Rolle Forschungsergebnissen aktuell zukommt. Die Fragen waren auch in diesem Leitfadenteil offen formuliert, was es den Lehrpersonen ermöglichen sollte, konkrete Handlungen und Situationen aus ihrer Praxis zu schildern - sofern sie dies jeweils als relevant ansahen (vgl. Nohl 2017, S. 16-19).

Die Datenauswertung erfolgte mit der Dokumentarischen Methode. Zunächst wurde im Zuge der formulierenden Interpretation der thematische Gehalt der vollständig transkribierten Interviews analysiert, indem das Gesagte paraphrasiert und zu Themen gebündelt wurde. Daraufhin erfolgte im Zuge der reflektierenden Interpretation ein Wechsel der Analyseeinstellung hin zu der Frage, wie etwas gesagt wurde. Grundlegend ist dabei die Annahme, dass sich die performative Logik erst durch eine „Einklammerung des Geltungscharakters“ (Mannheim 1980, S. 88, zit. n. Bohnsack 2017, S. 45) im Sinne einer „Suspendierung der Frage nach der faktischen Wahrheit oder normativen Richtigkeit des propositionalen Gehalts einer Äußerung oder Handlung“ (Bohnsack 2017, S. 77) erschließen lässt. Zur Unterscheidung von propositionaler und performativer Logik kam eine Textsortentrennung (vgl. Nohl 2017, S. 23 f., 32-35) zum Einsatz. Während sich Common Sense-Theorien und Normen aus Argumentationen (alltagstheoretische Zusammenfassungen) und Bewertungen (evaluative Stellungnahmen) herausarbeiten ließen, wurden die handlungsleitenden Habitus aus Erzählungen (Handlungs- und Geschehensabläufe mit zeitlicher Verortung), ergänzend dazu auch aus Beschreibungen (immer wiederkehrende Handlungsabläufe), rekonstruiert.

Erzählende (und beschreibende) Textpassagen wurden dabei einer ,komparative[n] Sequenzanalyse“ unterzogen, mittels derer die ,implizite Regelhaftigkeit“ (Nohl 2017, S. 36) bestimmt wurde, die ,aufeinander folgende[n] Erzählabschnitte strukturiert“ (Nohl 2017, S. 8). Dabei zeigte sich, dass dieselben Handlungsprobleme von den Fällen teilweise auf homologer, teilweise auf heterologer Weise erzählt bzw. beschrieben wurden. Über die Analyse minimaler und maximaler Kontraste ließen sich verschiedene Habitus im Sinne von Orientierungsrahmen (s. Abschn. 3) rekonstruieren. Im Vergleich mit anderen Fällen kristallisierten sich dabei jene negativen und positiven Gegenhorizonte heraus, von denen sich die Lehrpersonen handlungspraktisch distanzieren bzw. denen sie handlungspraktisch folgen (vgl. Bohnsack 2014, S. 137 f.).

Durch eine fortgesetzte komparative Analyse wurden daraufhin jene Aspekte der Habitus rekonstruiert, die sich von den einzelnen Fällen abstrahieren lassen, sich also fallübergreifend zeigen. Als gemeinsames Bezugsproblem, von dem in allen Fällen erzählt wird, stellte sich dabei die Gestaltung der unterrichtlichen Interaktion mit SchülerInnen unter den Aspekten Heteronomie und Autonomie heraus (s. Abschn. 5). Diese Basistypik wurde spezifiziert, indem durch eine Suche nach Kontrasten in der Gemeinsamkeit unterschiedliche Habitus der Bewältigung dieses Handlungsproblems identifiziert wurden (vgl. Bohnsack 2014, S. 143 ff.). In einem abschließenden Analyseschritt wurde gefragt, in welchem Verhältnis die auf diese Weise generierten sinngenetischen Typen zu der von den Lehrpersonen dargestellten Nutzung von Forschungsergebnissen stehen. 


\section{Ergebnisse}

Im Sample findet sich kein Fall, der bei den eingangs gestellten Interviewfragen bezüglich der Darstellung des Berufsalltages und der Schilderung von Unterrichtssituationen auf Forschungsergebnisse rekurriert. Stattdessen liegen Aussagen über die Nutzung von Forschungsergebnissen erst in jenen Interviewpassagen vor, in denen die Lehrpersonen auf diese Thematik angesprochen wurden. Diese Aussagen bestehen aus Argumentationen und Bewertungen, die Common Sense-Theorien der Lehrpersonen über die Nutzung von Forschungsergebnissen zum Ausdruck bringen (Ebene der propositionalen Logik). Die Habitus der Lehrpersonen bezüglich der Gestaltung des Berufsalltages lassen sich im Gegensatz dazu primär aus Erzählungen rekonstruieren, die zu Interviewbeginn verortet sind (Ebene der performativen Logik). Dabei zeigt sich, dass diese Habitus auch den Blick der Lehrpersonen auf Forschungsergebnisse und deren argumentative bzw. bewertende Auseinandersetzung mit diesen im Interview strukturieren.

Im Folgenden werden die Ergebnisse zunächst im Überblick dargestellt, ehe anhand zweier kontrastiver Fallbeispiele dargelegt wird, wie bestimmte Relationen von Common Sense-Theorien und Habitus eine Relevanzsetzung von Forschungsergebnissen verhindern bzw. ermöglichen. Bei den Ergebnissen im Überblick werden Zusammenfassungen präsentiert, die es ermöglichen sollen, die Fallbeispiele in einen größeren Kontext einzuordnen.

\subsection{Common Sense-Theorien und Habitus im Überblick}

Die Common Sense-Theorien über die Nutzung von Forschungsergebnissen bilden Alltagsinterpretationen der Lehrpersonen ab. Es wurden acht Orientierungsschemata herausgearbeitet, die auf Ebene des kommunikativen Wissens beschreiben, was die Interviewten unter Forschungsergebnissen fassen und wie sie diese nutzen:

- Individuelle Rezeption - Forschungsergebnisse werden von Lehrpersonen im Medium von Zeitschriften (etwa in Bildungsteilen), Fachliteratur (etwa zur Veränderung von Unterricht) und Büchern (etwa von Jesper Juul und Remo Largo) oder vermittelt durch Fortbildungen individuell rezipiert;

- Kollegiale Auseinandersetzung - Forschungsergebnisse dienen Lehrpersonen im Rahmen von Datenrückmeldungen (etwa im Zuge von Lernstandserhebungen) als Grundlage gemeinsamer Auseinandersetzungen an der Schule;

- Eigenes Forschen - Forschungsergebnisse werden von Lehrpersonen durch eigenes Forschen generiert, wobei zwischen zurückliegendem Forschen im Studium (etwa Recherchieren für Hausarbeiten, Labortätigkeit) und gegenwärtigem Forschen im Berufsalltag (etwa Durchführen unterrichtlicher Evaluationen, Vergleich von Fachcurricula für die Erstellung eines Schulbuches) unterschieden wird;

- Studienteilnahme - Forschungsergebnisse werden durch das Zutun der Lehrpersonen als Erforschte (etwa im Rahmen von Umfragen und studentischer Forschungsarbeiten) durch Externe generiert; 
- Grundhaltung - Forschungsergebnisse werden als Erkenntnisquelle angesehen, die von Lehrpersonen im Sinne einer forschenden Grundhaltung, die Reflexion von Alltagsroutinen ermöglicht, genutzt werden sollte;

- Unterrichtsnutzung - Forschungsergebnisse werden von Lehrpersonen als Unterrichtsgegenstand genutzt (etwa Forschungsergebnisse aufgreifen und Aufgaben daraus gestalten);

- SchülerInnenforschung - Forschungsergebnisse werden von SchülerInnen im Unterricht generiert (etwa im Rahmen Forschenden Lernens).

- Keine Relevanz - Forschungsergebnisse werden von Lehrpersonen explizit nicht genutzt.

Die rekonstruierten Habitus strukturieren auf Ebene des handlungsleitenden Wissens als Orientierungsrahmen, wie die Lehrpersonen mit Handlungsproblemen in ihrem Berufsalltag umgehen. Als allen Fällen gemeinsames Bezugsproblem hat sich im Zuge der Interpretation die Gestaltung der unterrichtlichen Interaktion unter den Aspekten Heteronomie und Autonomie herausgestellt. Aus Erzählungen über ihr eigenes Handeln wird deutlich, wie alle Lehrpersonen daran arbeiten, Unterrichtssettings herzustellen, die mit unterschiedlichen Ausprägungen von Heteronomie - verstanden als Fremdbestimmung der SchülerInnen durch die Lehrpersonen - und Autonomie - verstanden als den SchülerInnen von den Lehrpersonen gewährte Selbstbestimmung - verbunden sind. Andere Aspekte wie etwa die Auseinandersetzung mit der im Unterricht verhandelten Sache oder die Kooperation mit anderen Professionen scheinen hingegen nur vereinzelt im Datenmaterial auf. Mittels fortgesetzter Komparation wurden drei Typen gebildet, die beschreiben, wie die Lehrpersonen die unterrichtliche Interaktion mit SchülerInnen unter den Aspekten Heteronomie und Autonomie gestalten:

- Heteronomie auf Klassenebene - Umsetzung eines von der Lehrperson initiierten und gesteuerten gemeinsamen Unterrichts für alle, in dem die SchülerInnen keine Räume zur Selbstbestimmung erhalten;

- Heteronomie und Einzelfallorientierung - Umsetzung eines von der Lehrperson initiierten und gesteuerten Unterrichts, in dem die Lehrperson fallorientiert und unterstützend auf einzelne SchülerInnen eingeht;

- Heteronomie und partielle Autonomie - Umsetzung eines von der Lehrperson initiierten und gesteuerten Unterrichts, innerhalb dessen SchülerInnen partiell ermöglicht wird Entscheidungen hinsichtlich ihres Lernens zu treffen.

Im Folgenden wird anhand zweier kontrastiver Fälle exemplarisch aufgezeigt, in welchen Relationen sich Common Sense-Theorien über die Nutzung von Forschungsergebnissen und der Habitus der Gestaltung der unterrichtlichen Interaktion mit SchülerInnen zueinander befinden. 


\subsection{Falldarstellungen}

\subsubsection{Frau Otten: „Wir müssen erstmal lernen, wie es überhaupt gehen kann“}

Der Fall der Gymnasiallehrerin Frau Otten zeigt exemplarisch, wie die Beschäftigung mit Forschung Relevanz erhalten kann.

Der Habitus der Lehrerin bezüglich der Gestaltung der unterrichtlichen Interaktion mit SchülerInnen dokumentiert sich erstmals in der Erzählung einer erinnerten Unterrichtssituation:

englisches Theater, fünfte Klasse. (.) Ähm (...) wo es darum ging, dass die Schüler, nachdem geklärt war, wer welche Rolle hat, ähm in an verschiedenen Orten hier auf dem Schulhof [...] ähm üben sollten. Und ich merkte, dass fast zu jeder Gruppe, zu der ich dann so kontrollweise kam, ähm entweder einer die Situation komplett für sich genutzt hat, um da also nicht konkret zu arbeiten, sondern ganz was anderes zu machen [...] oder geschaukelt wurde, oder, oder. Jedenfalls gab es so viele Punkte, dass ich gesagt habe: ,So jetzt gehen wir alle wieder zusammen. Ähm ich habe gemerkt, dass ihr das in dieser Arbeitsform noch nicht könnt. Wir müssen jetzt erstmal hier ganz eng beieinanderbleiben, sodass ich das alles kontrollieren kann. [...] Wir müssen erstmal lernen, wie es überhaupt gehen kann" (Z. 106-121).

Diese Darstellung lässt sich in drei Sequenzen unterteilen. Zunächst führt Frau Otten dadurch in die Situation ein, dass sie Kontextinformationen zum Unterrichtsthema, zur Klassenstufe und zum Unterrichtssetting (Ort, Arbeitsaufgaben) nennt. Erkennbar wird dabei, dass sich die SchülerInnen in einem von der Lehrerin gesetzten Rahmen befinden, denn der Arbeitsauftrag, ein Theaterstück in Gruppen üben zu ,sollen', wird von der Lehrerin vorgegeben. Damit richtet die Lehrerin die Erwartung an die SchülerInnen, sich auf eine bestimmte Weise mit einem gegebenen Unterrichtsgegenstand auseinanderzusetzen (Sequenz 1). Daraufhin wird in der zweiten Sequenz deutlich, dass Frau Otten es nicht bei der Generierung dieses Rahmens belässt, sondern das Agieren der SchülerInnen dadurch ,kontrollweise“ erfasst, dass sie sich den Gruppen nähert und diese beobachtet (Sequenz 2). Als drittes schildert Frau Otten, wie sie qua verbaler Adressierung aller SchülerInnen auf ihre Beobachtungen reagiert hat. Dabei zeigt sich, dass Frau Otten in die Aktionen der SchülerInnen interveniert, die bisherigen Tätigkeiten der SchülerInnen unter Verweis auf deren aktuell als mangelnd eingeschätzte Kompetenz (,,ich habe gemerkt, dass ihr das in dieser Arbeitsform noch nicht könnt“") abbricht und das von ihr initiierte Unterrichtssetting auflöst. In der Ankündigung eines neuen Vorgehens (,Wir müssen erstmal lernen, wie es überhaupt gehen kann“) reproduziert sich, dass die Lehrerin nicht nur das Unterrichtssetting, sondern auch Erwartungen vorgibt und Handlungsdruck aufbaut. Anders als hinsichtlich des Übens des Theaterstücks auf dem Schulhof zieht sie sich dabei durch die Nutzung des Personalpronomens erste Person Plural mit ein, wobei unklar bleibt, worauf das Indefinitpronomen ,es“, auf das sich das gemeinsame ,Lernen-Müssen“ bezieht, genau verweist. Allerdings deutet sich in der Interpretation anderer Passagen desselben Interviews an, dass hier eher eine Entwicklungsperspektive hinsichtlich der Erfüllung der Erwartungen der 
Lehrerin denn hinsichtlich eines relativ freien Bearbeitens von Arbeitsaufträgen auf dem Schulhof aufscheint (Sequenz 3).

Zusammengefasst dokumentiert sich in diesem Interviewabschnitt ein Habitus, der die eigenen Erwartungshaltungen im Unterricht durchzusetzen sucht und die SchülerInnen fremdbestimmt. Diese Fremdbestimmung betrifft sowohl die Unterrichtsthemen als auch die Art und Weise, wie sich die SchülerInnen mit diesen Themen beschäftigen sollen. Anders ausgedrückt: Bei Frau Otten steht die Umsetzung ihrer normativen Erwartungen, die sie an das Agieren der SchülerInnen im Unterricht heranträgt, im positiven Gegenhorizont. Wie auch aus anderen Passagen desselben Interviews deutlich wird, bildet sich in der Beobachtung ihres Unterrichts unter dem Gesichtspunkt, ob die SchülerInnen ihren Erwartungen gerecht werden oder nicht, ihr unterrichtsbezogener Habitus ab. In letztgenannten Fällen, wenn das SchülerInnenhandeln im negativen Gegenhorizont zu ihren Erwartungen steht, interveniert sie in die Aktionen der SchülerInnen und sucht dadurch den Unterrichtsverlauf zu korrigieren (Typus: Heteronomie auf Klassenebene).

Im weiteren Interviewverlauf zeigt sich, dass Frau Otten im Medium ihres unterrichtsbezogenen Habitus über die Nutzung von Forschungsergebnissen spricht. Es wird in den diesbezüglichen primär argumentativen Passagen kein eigenständiger Habitus der Auseinandersetzung mit Forschungsergebnissen erkennbar, stattdessen werden die Aussagen durch den Heteronomie fokussierenden Habitus strukturiert. Deutlich wird, dass Frau Otten Forschungsergebnissen dann Relevanz beimisst, wenn sie zur Gestaltung ihres Unterrichts im Sinne einer Fremdbestimmung der SchülerInnen beitragen. So führt sie auf die Leitfadenfrage nach der derzeitigen Rolle von Forschung für ihren Berufsalltag als erstes an, dass Forschungsergebnisse dann relevant seien, wenn sie ihrem Unterricht dienen (Common Sense-Theorie ,Unterrichtsnutzung“). Sie nutze „,neueste Forschungsergebnisse“ als Unterrichtsgegenstände ,immer, sofern es [...] zum Thema passt“ (Z. 505 f.). Es gehe ihr darum, die SchülerInnen ,damit zu ähm konfrontieren. Ähm war das schon immer so? Hat sich da was verändert? Hat sich verändert sich die Gesellschaft gerade eben im Moment?“ (Z. 509-511). Forschungsergebnisse werden dabei nicht als eigenständiges Unterrichtsthema gesehen, sondern als Ergänzung zum eigentlichen Thema. Die Lehrerin erscheint in dieser Passage, wie oben bereits herausgearbeitet, als Gestalterin des Unterrichts, die Unterrichtsthemen und den Umgang mit diesen setzt. Den SchülerInnen wird hingegen kollektiv die Rolle der Reagierenden zugewiesen, die sich als Konfrontierte mit dem von ihr Vorgegeben - hier Forschungsergebnissen auseinandersetzen sollen.

Das Analyseergebnis, dass die Lehrerin im Medium ihres Habitus über die Nutzung von Forschungsergebnissen spricht, bestätigt sich im weiteren Interviewverlauf, wenn Frau Otten auf die Frage nach der Rolle von Forschung für ihren Berufsalltag weiter ausführt, dass es an ihrer Schule „Forscherkurse“ (Z. 514) gibt. Diese Kurse werden - entsprechend ihres auf Heteronomie im Unterricht setzenden Habitus - als Unterrichtseinheiten eingeführt, in denen die Lehrpersonen ,,die Schüler dazu bringen ähm kritisch oder auch kreativ an bestimmte Fragen heranzugehen“ (Z. 517f.). Zwar erscheint Forschung in dieser und der folgenden Darstellung über die Forscherkurse als etwas, das von den SchülerInnen selber durchgeführt wird (Common Sense-Theorie ,SchülerInnenforschung'), doch zeigt sich in der einleitenden Wort- 
wahl des ,dazu bringen“, dass die SchülerInnen auch hier (zumindest zunächst) als Gegenüber angesehen werden, mit denen etwas gemacht wird bzw. die sich auf bestimmte, von den Lehrpersonen vorgegebene Weise verhalten sollen und in diesem Sinne fremdbestimmt werden.

Drittens spricht die Lehrerin im Rahmen ihrer Antwort über an der Schule stattfindende Lerncoach-Treffen. Auch hier deklariert Frau Otten Relevanz von Forschungsergebnissen und argumentiert, dass diese dann „wichtig“ (Z. 538) seien, ,wenn ich mit den Lerncoaches überlege: Wie kriegen wir die Schüler dazu motiviert zu sein? [...] Dann ist es ja interessant zu sehen: Was hat man überhaupt über Motivation herausgefunden?“ (Z. 545-549) (Common Sense-Theorie ,kollegiale Auseinandersetzung '). Die Auseinandersetzung mit Forschungsergebnissen wird als relevant die Bezeichnungen schwanken zwischen ,wichtig“ und ,,interessant“ - für die Unterstützung von Lernprozessen dargestellt. Gleichzeitig wird auch diese Aussage im Rahmen des Heteronomie auf Klassenebene fokussierenden Habitus getätigt, denn wiederum gehen die Aktionen von den Lehrpersonen aus, während die SchülerInnen als ununterscheidbare Gegenüber erscheinen, die sich im Unterricht auf bestimmte Weise, hier „motiviert“, zeigen sollen. Vor diesem Hintergrund erscheint die Einrichtung von ,Lerncoaches“ als von der Lehrerin mitgetragene Intervention, um ein erwünschtes SchülerInnenverhalten zu erreichen.

\subsubsection{Frau Schaller: „das große Feuer, was vor einem ist, irgendwie in Griff zu kriegen"}

Das Interview mit der Grundschullehrerin Frau Schaller steht für Fälle, die Forschung keinerlei Relevanz zuschreiben.

Wie Frau Otten kommt Frau Schaller dann ins Erzählen, wenn sie über ihren Unterricht spricht:

ich hab jetzt einen Sachunterrichtstest neulich geschrieben und hab den natürlich dreifach differenziert und trotzdem kam ein Schüler, das ist dieser Khalil, ähm mit dem Test überhaupt nicht zurecht, alleine, weil er einfach ganz große Probleme hat zu lesen. [...] Ich konnte während des Tests mich nicht neben ihn setzen und mit ihm den Test so zusammen durchgehen und besprechen, weil ich natürlich den Blick auf die ganze Klasse haben musste. Und am Ende, wo aber fast schon alle anderen Kinder abgegeben haben, da hatte ich Zeit zu Khalil zu gehen und dann haben wir, hab ich ihm die Aufgaben vorgelesen und er sollte bestimmte Wörter zusortieren und das hat er alles auch relativ gut hinbekommen (Z. 137-147).

Diese Darstellung einer erinnerten Unterrichtssituation weist eine vergleichbare Struktur wie die Darstellung aus dem Englischunterricht von Frau Otten auf. Zunächst führt die Lehrerin Rahmenbedingungen des Unterrichts an, die von ihr gesetzt wurden. Sprachlich wird dies daran deutlich, dass die Lehrerin den „Sachunterrichtstest“" nicht von den SchülerInnen durchführen ließ, sondern formuliert, diesen selber absolviert zu haben (,ich hab [...] geschrieben“). Damit zeigt sich als vergleichbares Thema, das als Tertium Comparationis den Vergleich zwischen den beiden Fällen strukturiert, dass die Rahmung der Unterrichtssituation von der 
jeweiligen Lehrerin vorgegeben wird (Sequenz 1). Wie Frau Otten berichtet auch Frau Schaller daraufhin davon, sich nach Setzung dieser Rahmung nicht aus der Situation zurückgezogen, sondern die SchülerInnen beobachtet zu haben, wie sie mit der vorgegebenen Anforderung umgehen. Anders als Frau Otten fokussiert die Lehrerin dabei aber nicht auf Gruppen beziehungsweise letztlich auf die gesamte Klasse, sondern auf einen Einzelschüler, „Khalil“, der sich zudem nicht wie die SchülerInnen im Fall Frau Otten Tätigkeiten gewidmet hat, die in den Augen der Lehrperson nicht zur Anforderung gehören. Stattdessen hatte der Schüler laut Interpretation Frau Schallers trotz einer vorgenommenen ,dreifachen Differenzierung ‘ des Tests „Probleme“, der gestellten Anforderung auf Grund seiner unzureichenden Lesekompetenz nachzukommen. Als normative Erwartung, deren Umsetzung im positiven Gegenhorizont steht, dokumentiert sich hier, dass die vorgenommene Differenzierung dazu führt, dass die SchülerInnen den Test problemfrei bearbeiten können (Sequenz 2). Als drittes thematisiert auch Frau Schaller, auf ihre Beobachtung reagiert zu haben, doch interveniert sie anders als Frau Otten nicht unmittelbar, sondern erst mit Verzögerung. Sie bricht das von ihr initiierte Unterrichtssetting dabei nicht $a b$, sondern modifiziert es dadurch, dass sie sich dem Einzelschüler zuwendet und ihn bei der Bearbeitung des Tests dadurch unterstützt, dass sie die konstatierten Leseprobleme des Schülers durch Vorlesen der Aufgabe situativ zu kompensieren sucht (Sequenz 3).

Es zeigt sich hier insgesamt, dass ein vergleichbares Bezugsproblem im Kontext der Gestaltung der unterrichtlichen Interaktion - SchülerInnen erfüllen nicht die unterrichtsbezogene Erwartung der Lehrperson - zwar sowohl bei Frau Otten als bei Frau Schaller im negativen Gegenhorizont steht, aber unterschiedlich bearbeitet wird. Während der Habitus von Frau Otten Heteronomie auf Klassenebene umzusetzen sucht, zeigt sich bei Frau Schaller ein Habitus der fallorientierten Unterstützung innerhalb eines Unterrichtssettings, das den SchülerInnen von der Lehrerin vorgegeben wird. Die Gesamtklasse gerät dabei bei Frau Schaller nicht aus dem Blick. Stattdessen wägt sie situativ ab, wann sie sich welchen EinzelschülerInnen zuwenden kann (Typus: Heteronomie und Einzelfallorientierung).

Auch bei Frau Schaller erfolgt die Auseinandersetzung mit Forschung im Medium ihres unterrichtsbezogenen Habitus. Während allerdings Frau Otten auf kommunikativer Ebene die Relevanz der Nutzung von Forschungsergebnissen herausgestellt hat, scheint der unterrichtsbezogene Habitus von Frau Schaller eine Relevanzsetzung von Forschungsergebnissen zu verschließen (Common Sense-Theorie: keine Relevanz). So antwortet die Lehrerin auf die Frage, ob Forschung für ihren Berufsalltag eine Rolle spielt, wie folgt:

Nein. [...] Dass ich mir irgendwelche Studien durchlese, nein. [...] Des ist eher so für mich is Berufsalltag ganz viel ähm also das das große Feuer, was vor einem ist, irgendwie in Griff zu kriegen. Und das heißt, ich beschäftige mich lieber damit, wie mache ich, dass Melanie sich aufhört mit David zu prügeln? Und da würde ich mir nicht irgendwelche Studien [...] zu durchlesen, sondern ähm da frag ich vielleicht ne Kollegin oder ich denk mir da selbst irgendwas aus, ähm dass ich mich mit denen nochmal hinsetze oder ob ich mit den Eltern, aber es ist nicht so, dass ich diesen ganz großen Blick ähm ,Gibts vielleicht irgendwelche Studien dazu?" oder so einnehme (Z. 403-412). 
Die Lehrerin grenzt ihre Handlungspraxis von der Lektüre von Studien ab und entwirft stattdessen das Bild, das große Feuer, was vor einem ist, irgendwie in den Griff zu kriegen'. Praxis ist demnach durch Unmittelbarkeit, Ungewissheit und einen Anforderungscharakter gekennzeichnet, denn sie verlangt nach fallspezifischer Intervention, wie die Nennung einer konkreten Auseinandersetzung zwischen zwei SchülerInnen verdeutlicht. Frau Schallers „Blick“ bleibt damit ihrer Darstellung nach auf die schulische Interaktion mit SchülerInnen und KollegInnen fokussiert, er wird - anders als gemäß der Darstellung von Frau Otten - nicht durch die Recherche und Lektüre von Forschungsergebnissen erweitert.

Es scheint insbesondere die Einzelfallorientierung zu sein, vor deren Hintergrund Frau Schaller Forschungsergebnisse als irrelevant ansieht. So führt die Lehrerin weiter aus:

im Radio kommen ja auch dann die neuen PISA-Ergebnisse und dann ähm kommt da irgendwie „Bremen hat wieder so schlecht abgeschnitten“ un- und was hilft mir das für meinen Lehreralltag? Was hilft mir das für meinen Schultag morgen? Was hilft mir das für mein Khalil-Problem? Für mein Josie-Problem? Es hilft mir im Grunde nichts. Ich versuche jeden Tag meinen Schülern den besten Unterricht zu geben, den ich von meiner Kraft her schaffe und den ich von meinem Wissen als den besten halte. Ähm (.) und (..) deswegen hör ich mir das im Radio an, aber ich zieh da keine Schlüsse au-au- auf auf meinen Unterricht oder ähm, nee (Z. 431-440).

Frau Schaller fügt ein Beispiel an:

also wenn rauskommt, ,immer weniger Kinder können äh sinnentnehmend lesen“. Dann gehe ich nicht am nächsten Tag in meine Klasse und äh sage, „oh, ihr könnt ja alle gar nicht sinnentnehmend lesen“. Ich weiß ja, wie meine Schüler alle sind (Z. 444-448).

Die Lehrerin assoziiert Forschung mit Ergebnissen einer internationalen Schulleistungsstudie und betrachtet diese unter der Fragestellung, inwiefern sie ihr bei der situativen Lösung ihrer spezifischen unterrichtsbezogenen Probleme mit EinzelschülerInnen und der Gestaltung ihres Unterrichts helfen könnten. Das Wissen, das sie durch die Rezeption solcher Forschungsergebnisse erhält, erscheint dabei auf Grundlage ihres auf Einzelfallorientierung innerhalb von ihr vorgegebener Unterrichtssettings fokussierenden Habitus nicht kompatibel mit ihrem eigenen, routinisierten Wissen um die Gestaltung ihres Unterrichts (,,jeden Tag [...] Unterricht geben“) und um das Können ihrer SchülerInnen (,wie meine Schüler alle sind“).

\subsection{Fazit}

Resümierend ist mit Blick auf die Forschungsfrage festzuhalten, dass hinsichtlich der Nutzung von Forschungsergebnissen kommunikative Wissensbestände im Sinne von Common Sense-Theorien relevant werden. Handlungsleitende Wissensbestände zeigen sich indes in Abgrenzung dazu in Habitus bezüglich der Gestaltung der unterrichtlichen Interaktion mit SchülerInnen. Dabei dokumentieren sich folgende Relationen: Bei Frau Otten lässt sich erkennen, dass mehrere Common Sense- 
Theorien von der Lehrerin im Medium ihres Habitus wahrgenommen werden. Die Lehrerin entfaltet diese Theorien vermittelt durch ein typisches Wissen um die Gestaltung von Unterricht, konkret vermittelt durch einen auf Heteronomie fokussierenden Habitus. Ihr unterrichtsbezogener Habitus eröffnet es ihr dabei, verschiedene Relevanzsetzungen von Forschungsergebnissen für die Gestaltung ihres Unterrichts wahrzunehmen. Auch bei Frau Schaller wurde ein auf Heteronomie fokussierender Habitus rekonstruiert, der allerdings darüber hinaus ein fallorientiertes Eingehen auf EinzelschülerInnen umfasst. Ebenso wie Frau Otten nimmt diese Lehrerin Forschungsergebnisse im Medium ihres Habitus wahr, allerdings kommt es bei ihr anders als bei Frau Otten - zu einer Irrelevanzsetzung von Forschungsergebnissen. Dabei scheint es insbesondere die Einzelfallorientierung im Unterricht zu sein, die sich bei der Lehrerin als nicht kompatibel mit einer Nutzung von Forschungsergebnissen erweist.

\section{Diskussion}

Aufbauend auf mittels standardisierter und inhaltsanalytischer Forschung generierten Befunden wurde ein explorativer Weg präsentiert, wie sich die vielfach beschriebene eher geringe Nutzung von Forschungsergebnissen durch Lehrpersonen vertiefend analysieren lässt. Bezüglich der Forschungsfrage, welche Bedeutung kommunikativen und handlungsleitenden Wissensbeständen von Lehrpersonen bei der Nutzung von Forschungsergebnissen zukommt, zeigte sich, dass die interviewten Lehrpersonen ausschließlich auf kommunikatives Wissen zurückgreifen. Dieses Ergebnis deckt sich insofern mit dem Forschungsstand (s. Abschn. 2), als die Beschäftigung mit Forschungsergebnissen in den Interviews auf der Ebene von Common SenseTheorien verblieb und nicht die Ebene von Habitus als Modi Operandi der beruflichen Praxis erreichte.

Darüber hinausgehend wurde deutlich, dass die kommunikativen von den habituellen Wissensbeständen gerahmt sind, d.h., dass die kommunizierte Auseinandersetzung mit Forschungsergebnissen sowohl im Fall ihrer Relevanz- (etwa Fall Frau Otten) als auch ihrer Irrelevanzsetzung (etwa Fall Frau Schaller), ,im Medium ihres Habitus“ (Sotzek et al. 2017, S. 317) erfolgt: Forschungsergebnisse werden von den interviewten Lehrpersonen vermittelt durch ihren Habitus wahrgenommen, also unter der Perspektive ihres handlungspraktischen Umgangs mit unterrichtsbezogenen Problemen. Jenes Wissen, das ihr unterrichtsbezogenes Handeln strukturiert, liegt auch dem Sprechen über Forschungsergebnisse zugrunde, es bildet die Brille, mit der die Lehrpersonen auf Forschungsergebnisse blicken. Anders ausgedrückt wird die Auseinandersetzung mit Forschungsergebnissen erst durch den Habitus der Lehrpersonen „gerahmt“, d.h. ,integriert oder gebrochen“ (Sotzek et al. 2017, S. 319; vgl. auch Bohnsack 2017, S. 107f.). Daraus ist aber angesichts des exemplarischen Vorgehens der Studie nicht zu folgern, dass beispielsweise jegliche Einzelfallorientierung zu einer Irrelevanzsetzung von Forschungsergebnissen führen würde. Vielmehr soll die Analyse am Beispiel zweier Fälle veranschaulichen, dass die Art und Weise, wie Lehrpersonen über die Nutzung von Forschungsergebnissen sprechen, nicht losgelöst von ihrem unterrichtsbezogenen Habitus, sondern im Medium dieses 
Habitus erfolgt. Es bedürfte Untersuchungen mit größeren Fallzahlen, um nähere Aussagen über die Bedeutung des Auftretens bestimmter Common Sense-Theorien im Medium bestimmter Habitus treffen zu können. Eine weitere Frage für künftige Forschung lautet, inwiefern die unterrichtsbezogenen Habitus der Lehrpersonen, auch jene, die mit einer Irrelevanzsetzung von Forschungsergebnissen einhergehen, in ihrer Genese, etwa im Studium und im Referendariat, durch die Nutzung von Forschungsergebnissen geprägt worden sind.

Die Studie verweist darauf, dass Lehrpersonen habituell an der Gestaltung der unterrichtlichen Interaktion mit SchülerInnen arbeiten - ein Ergebnis, das im Diskurs um LehrerInnenhandeln nicht überrascht (vgl. etwa Bonnet und Hericks 2014; KMK 2019). Spezifizierend zeigte sich, dass zumindest die befragten Lehrpersonen bei der Gestaltung ihres Unterrichts SchülerInnen fremdbestimmen, wobei unterschiedliche Typen sichtbar wurden (vgl. auch Helsper und Lingkost 2004). Die rekonstruierten Habitus beschäftigen sich dabei nicht mit der von Seiten der Bildungspolitik, -administration und Teilen der Bildungsforschung herangetragenen Erwartung, Forschungsergebnisse zu nutzen (s. Abschn. 1). Für eine Förderung der handlungspraktischen Bedeutung von Forschungsergebnissen wäre somit weniger die Betonung der normativen Setzung, sich mit Forschungsergebnissen auseinandersetzen zu sollen, weiterführend als vielmehr die Überlegung, wie diese Norm durch die unterrichtsbezogenen Habitus der Lehrpersonen gerahmt wird.

Anschlüsse zeigen sich hier zu Studien, die aufbauend auf der Diagnose eines Technologiedefizits im LehrerInnenhandeln eine „technokratische Logik“ (van Ackeren et al. 2017, S. 254) kritisieren, welche mit dem Steuerungsmodell der Evidenzbasierung verbunden wird (vgl. etwa Herzog 2016). Die durchgeführte Studie bestätigt Forschungsergebnisse, nach denen eine Anwendung von Forschungsergebnissen im Sinne einer Ableitung von Handeln aus Regeln an der Praxis von Lehrpersonen vorbeigeht und eine darauf basierende Implementations- und Transferstrategie letztlich nicht erfolgreich sein kann (vgl. etwa Hartmann et al. 2016). Eine einfache Übernahme von externen Informationen ist nicht möglich - so auch ein zentrales Ergebnis der Wissensverwendungsforschung (vgl. etwa Altrichter 2019).

Kritisch anzumerken ist, dass in der durchgeführten Studie Habitus im Sinne einer ,proponierten Performanz“ untersucht wurden, während die ,performative Performanz“ (Bohnsack 2017, S. 93) der Lehrpersonen, die sich aus der Beobachtung der Praxis von Lehrpersonen bspw. via Unterrichtsvideographien (vgl. etwa Asbrand und Martens 2018) herausarbeiten ließe, nicht analysiert wurde. Die praktische Umsetzung von handlungsleitenden Orientierungen wurde somit nicht erfasst. Eine weitere Grenze besteht darin, dass auf Grund des Analyseaufwands nur relativ kleine Fallzahlen einbezogen werden konnten. Die Übertragbarkeit der Ergebnisse ist indes durch die Verankerung der Studie in der Praxeologischen Wissenssoziologie insofern potenziell gegeben, als sich die Unterscheidung von kommunikativem und handlungsleitendem Wissen auch in anderen Studien zur Erschließung des Berufsalltages von Lehrpersonen als tragfähig erwiesen hat (vgl. etwa Hinzke 2018; Sotzek et al. 2017).

Insgesamt betrachtet bietet die durchgeführte Studie Erklärungsansätze dafür, warum Lehrpersonen Forschungsergebnisse als unterschiedlich relevant einschätzen und selektiv wahrnehmen (s. Abschn. 2). Sie kann darüber hinaus als Beitrag zum 
aktuellen Diskurs um die Erfassung eines „Lehrerhabitus“ (Helsper 2018) verstanden werden. Die analysierten Habitus beziehen sich dabei auf das berufspraktische Handlungsproblem, die unterrichtliche Interaktion mit SchülerInnen zu gestalten, und lassen sich insofern als berufliche Habitus oder präziser - der prinzipiellen Mehrdimensionalität der Typenbildung in der Praxeologischen Wissenssoziologie Rechnung tragend (vgl. Bohnsack 2017, S. 117-120) - als eine Dimension eines LehrerInnenhabitus bezeichnen. Als solche drücken sich in ihnen „fachliche und pädagogische Praxen der jeweiligen Lehrertätigkeit“" (Helsper 2018, S. 123) aus und es wird ersichtlich, dass sie sich in Relation zu Normen und Common Sense-Theorien entfalten (vgl. auch Hericks et al. 2018). Weitere Studien mit größeren Samples könnten Ergebnisse dazu generieren, in welcher Relation die beruflichen Habitus, aber auch die Common Sense-Theorien über die Nutzung von Forschungsergebnissen zur Schulform und Berufsbiographie sowie ggf. auch zu jener Schulkultur (vgl. etwa Böhme et al. 2015) stehen, innerhalb derer Lehrpersonen unterrichten.

Funding Open Access funding enabled and organized by Projekt DEAL.

Open Access Dieser Artikel wird unter der Creative Commons Namensnennung 4.0 International Lizenz veröffentlicht, welche die Nutzung, Vervielfältigung, Bearbeitung, Verbreitung und Wiedergabe in jeglichem Medium und Format erlaubt, sofern Sie den/die ursprünglichen Autor(en) und die Quelle ordnungsgemäß nennen, einen Link zur Creative Commons Lizenz beifügen und angeben, ob Änderungen vorgenommen wurden.

Die in diesem Artikel enthaltenen Bilder und sonstiges Drittmaterial unterliegen ebenfalls der genannten Creative Commons Lizenz, sofern sich aus der Abbildungslegende nichts anderes ergibt. Sofern das betreffende Material nicht unter der genannten Creative Commons Lizenz steht und die betreffende Handlung nicht nach gesetzlichen Vorschriften erlaubt ist, ist für die oben aufgeführten Weiterverwendungen des Materials die Einwilligung des jeweiligen Rechteinhabers einzuholen.

Weitere Details zur Lizenz entnehmen Sie bitte der Lizenzinformation auf http://creativecommons.org/ licenses/by/4.0/deed.de.

\section{Literatur}

van Ackeren I., Binnewies, C., Clausen, M., Demski, D., Dormann, C., Koch, A.R., Laier, B., Preisendörfer, P., Preuße, D., Rosenbusch, C., Schmidt, U., Stump, M., \& Zlatkin-Troitschanskaia, O. (2013). Welche Wissensbestände nutzen Schulen im Kontext von Schulentwicklung? In I. van Ackeren, M. Heinrich \& F. Thiel (Hrsg.), Evidenzbasierte Steuerung im Bildungssystem? (DDS: Beiheft 12, S. 51-73). Münster: Waxmann.

van Ackeren, I., Demski, D., \& Klein, E. D. (2017). Entwicklungsprobleme Neuer Steuerung im Schulsystem. In H. G. Holtappels (Hrsg.), Entwicklung und Qualität des Schulsystems (S. 241-259). Münster: Waxmann.

Altrichter, H. (2019). Transfer ist Arbeit und Lernen. In C. Schreiner, C. Wiesner, S. Breit, P. Dobbelstein, M. Heinrich \& U. Steffens (Hrsg.), Praxistransfer Schul- und Unterrichtsentwicklung (S. 27-33). Münster: Waxmann.

Altrichter, H., \& Merki, M. K. (2016). Steuerung der Entwicklung des Schulwesens. In H. Altrichter \& K. Maag Merki (Hrsg.), Handbuch Neue Steuerung im Schulsystem (S. 1-27). Wiesbaden: Springer VS.

Altrichter, H., Moosbrugger, R., \& Zuber, J. (2016). Schul- und Unterrichtsentwicklung durch Datenrückmeldung. In H. Altrichter \& K. Maag Merki (Hrsg.), Handbuch Neue Steuerung im Schulsystem (S. 235-277). Wiesbaden: Springer VS.

Asbrand, B., \& Martens, M. (2018). Dokumentarische Unterrichtsforschung. Wiesbaden: Springer VS. 
Bach, A., Wurster, S., Thillmann, K., Pant, H. A., \& Thiel, F. (2014). Vergleichsarbeiten und schulische Personalentwicklung. Zeitschrift für Erziehungswissenschaft, 17(1), 61-84.

Bauer, J., Prenzel, M., \& Renkl, A. (2015). Evidenzbasierte Praxis - im Lehrerberuf?! Unterrichtswissenschaft, 43(3), 188-192.

Böhme, J., Hummrich, M., \& Kramer, R.-T. (Hrsg.). (2015). Schulkultur. Wiesbaden: Springer VS.

Bohnsack, R. (2014). Rekonstruktive Sozialforschung (9. Aufl.). Opladen: Budrich.

Bohnsack, R. (2017). Praxeologische Wissenssoziologie. Opladen: Budrich.

Bonnet, A., \& Hericks, U. (2014). Professionalisierung und Deprofessionalisierung im Lehrer/innenberuf. Zeitschrift für Interpretative Schul- und Unterrichtsforschung, 3, 3-8.

Bonsen, M., \& Berkemeyer, N. (2014). Lehrerinnen und Lehrer in Schulentwicklungsprozessen. In E. Terhart, H. Bennewitz \& M. Rothland (Hrsg.), Handbuch der Forschung zum Lehrerberuf (2. Aufl., S. 920-936). Münster: Waxmann.

Bromme, R., Prenzel, M., \& Jäger, M. (2014). Empirische Bildungsforschung und evidenzbasierte Bildungspolitik. In R. Bromme \& M. Prenzel (Hrsg.), Von der Forschung zur evidenzbasierten Entscheidung (Zeitschrift für Erziehungswissenschaft: Sonderheft 27, S. 3-54). Wiesbaden: Springer VS.

Demski, D. (2017). Evidenzbasierte Schulentwicklung. Wiesbaden: Springer VS.

Demski, D., van Ackeren, I., \& Clausen, M. (2016). Zum Zusammenhang von Schulkultur und evidenzbasiertem Handeln. Journal for Educational Research Online, 8(3), 39-58.

Flick, U. (2017). Qualitative Sozialforschung. Eine Einführung (8. Aufl.). Reinbek: Rowohlt.

Hartmann, U., Decristan, J., \& Klieme, E. (2016). Unterricht als Feld evidenzbasierter Bildungspraxis? In J. Baumert \& K.-J. Tillmann (Hrsg.), Empirische Bildungsforschung (Zeitschrift für Erziehungswissenschaft: Sonderheft 31, S. 179-199). Wiesbaden: Springer VS.

Helsper, W. (2018). Lehrerhabitus. In A. Paseka, M. Keller-Schneider \& A. Combe (Hrsg.), Ungewissheit als Herausforderung für pädagogisches Handeln (S. 105-140). Wiesbaden: Springer VS.

Helsper, W., \& Lingkost, A. (2004). Schülerpartizipation in den Antinomien modernisierter Schulkultur. In W. Helsper, M. Kamp \& B. Stelmaszyk (Hrsg.), Schule und Jugendforschung zum 20. Jahrhundert (S. 198-230). Wiesbaden: VS.

Hericks, U., Sotzek, J., Rauschenberg, A., Wittek, D., \& Keller-Schneider, M. (2018). Habitus und Normen im Berufseinstieg von Lehrer*innen - eine mehrdimensionale Typenbildung aus der Perspektive der Dokumentarischen Methode. Zeitschrift für Interpretative Schul- und Unterrichtsforschung, 7, 65-80.

Herzog, W. (2016). Kritik der evidenzbasierten Pädagogik. In J. Baumert \& K.-J. Tillmann (Hrsg.), Empirische Bildungsforschung (Zeitschrift für Erziehungswissenschaft: Sonderheft 31, S. 201-213). Wiesbaden: Springer VS.

Hetmanek, A., Wecker, C., Kiesewetter, J., Trempler, K., Fischer, M. R., Gräsel, C., \& Fischer, F. (2015). Wozu nutzen Lehrkräfte welche Ressourcen? Unterrichtswissenschaft, 43(3), 193-208.

Hinzke, J.-H. (2018). Lehrerkrisen im Berufsalltag. Zum Umgang mit Spannungen zwischen Normen und Orientierungsrahmen. Wiesbaden: Springer VS.

KMK (2016). Gesamtstrategie der Kultusministerkonferenz zum Bildungsmonitoring. Köln: Carl Link.

KMK (2019). Standards für die Lehrerbildung: Bildungswissenschaft. Berlin. https://www.kmk.org/ fileadmin/veroeffentlichungen_beschluesse/2004/2004_12_16-Standards-Lehrerbildung-Bildungs wissenschaften.pdf. Zugegriffen: 14. Okt. 2020.

Kuper, H., Maier, U., Graf, T., Muslic, B., \& Ramsteck, C. (2016). Datenbasierte Schulentwicklung mit Vergleichsarbeiten aus der Perspektive von Lehrkräften, Fachkonferenzleistungen, Schulleitungen und Schulaufsichten. In BMBF (Hrsg.), Steuerung im Bildungssystem (S. 39-67). Berlin: BMBF. https://www.bmbf.de/upload_filestore/pub/Bildungsforschung_Band_43.pdf. Zugegriffen: 14. Okt. 2020 .

Landesinstitute und Qualitätseinrichtungen (2018). Positionspapier der Landesinstitute und Qualitätseinrichtungen der Länder zum Transfer von Forschungswissen. Soest: Landesinstitute und Qualitätseinrichtungen.

Nohl, A.-M. (2017). Interview und Dokumentarische Methode (5. Aufl.). Wiesbaden: Springer VS.

Otto, J., Hinzke, J.-H., \& Besa, K.-S. (2019). Wozu beschäftigen sich Lehrpersonen mit Forschung? Erste Ergebnisse aus dem Projekt NuBiL (Nutzung von Bildungsforschung durch Lehrpersonen). In V. Manitius \& N. van Holt (Hrsg.), Transfer zwischen Lehrer(fort)bildung und Wissenschaft (S. 71-88). Bielefeld: WBV.

Rochnia, M., \& Trempler, K. (2019). Welche externen Wissensquellen bevorzugen Lehrkräfte für ihr professionelles Handeln? Lehrerbildung auf dem Prüfstand, 12(2), 125-142.

Sotzek, J., Wittek, D., Rauschenberg, A., Hericks, U., \& Keller-Schneider, M. (2017). Spannungsverhältnisse im Berufseinstieg von Lehrpersonen. Zeitschrift für Qualitative Forschung, 18(2), 315-333. 
Terhart, E. (2011). Lehrerberuf und Professionalität. In W. Helsper \& R. Tippelt (Hrsg.), Pädagogische Professionalität (Zeitschrift für Pädagogik: Beiheft 57, S. 202-224). Weinheim: Beltz.

Thiel, F., Tarkian, J., Lankes, E.-M., Maritzen, N., \& Riecke-Baulecke, T. (2019). Strategien datenbasierter Steuerung zur Sicherung und Entwicklung von Schulqualität in den 16 Ländern. In F. Thiel, J. Tarkian, E.-M. Lankes, N. Maritzen, T. Riecke-Baulecke \& A. Kroupa (Hrsg.), Datenbasierte Qualitätssicherung und -entwicklung in Schulen (S. 313-325). Wiesbaden: Springer VS.

Wurster, S., Richter, D., \& Lenski, A. E. (2017). Datenbasierte Unterrichtsentwicklung und ihr Zusammenhang zur Schülerleistung. Zeitschrift für Erziehungswissenschaft, 20(4), 628-650.

Zlatkin-Troitschanskaia, O. (2016). Evidence-based actions with the multilevel system of schools. Journal for Educational Research Online, 8(3), 5-13.

Zlatkin-Troitschanskaia, O., Zimmer, L. M., Mater, O., Laier, B., Koch, A. R., Binnewies, C., Dormann, C., van Ackeren, I., Clausen, M., Preisendörfer, P., Schmidt, U., Demski, D., Preuße, D., \& Stump, M. (2016). Schulische und individuelle Einflussfaktoren auf das evidenzbasierte Handeln von Lehrkräften und Schulleitungen. In BMBF (Hrsg.), Steuerung im Bildungssystem (S. 8-38). Berlin: BMBF. https://www.bmbf.de/upload_filestore/pub/Bildungsforschung_Band_43.pdf. Zugegriffen: 14. Okt. 2020 . 